\title{
The study of capacity and installation of distributed power flow
}

\section{controller}

\author{
Menglu Gao ${ }^{1, a}$, Qianguang Yuan ${ }^{1, b}$, Aihong Tang ${ }^{1, c}$ \\ ${ }^{1}$ School of Automation, Wuhan University of technology, Wuhan, Hubei, 430070, China \\ a790016332@qq.com, b251187806@qq.com, c375943243@qq.com
}

\begin{abstract}
Keywords: Distributed power flow controller, system parameters, capacity, installation location Abstract. This paper deals with the capacity and installation problems of the Distributed Power Flow Controller. There is a rapidly increase in the development of FACTS and the application of it in the power grid. On $500 \mathrm{kV}$ power distributed system, the addition of Distributed Power Flow Controller is to change the line parameters, but the capacity of the Distributed Power Flow Controller adding into the system and how to install on the line are the primary problems need to be solved. In this paper, we study the best location as well as the appropriate capacity of the installation of Distributed Power Flow Controller, and launch the foundation of practical application for the actual project.
\end{abstract}

\section{Introduction}

In 2007, Yuan Zhihui, who studied in the Holland Delft University of science and technology, proposed the concept of distributed power flow controller (DPFC) [1].

DPFC is designed with the thought, adopting distributed static series compensation, in order to distribute the three-phase converter which has large capacity of series side of UPFC. While small capacity single-phase converters adopt decentralized articulated in the transmission line. The third harmonic is used to exchange the active power from parallel side to series side so that the power flow of the power system could be regulated comprehensively and this behavior is based on the new circuit topology. DPFC is used to control or regulate the bus voltage and the active power and reactive power of the transmission lines dynamically. The DPFC consists of converters of the parallel side, converter of the series sides, the pathway of the third harmonic, and the simplified schematic is shown in Figure 1.

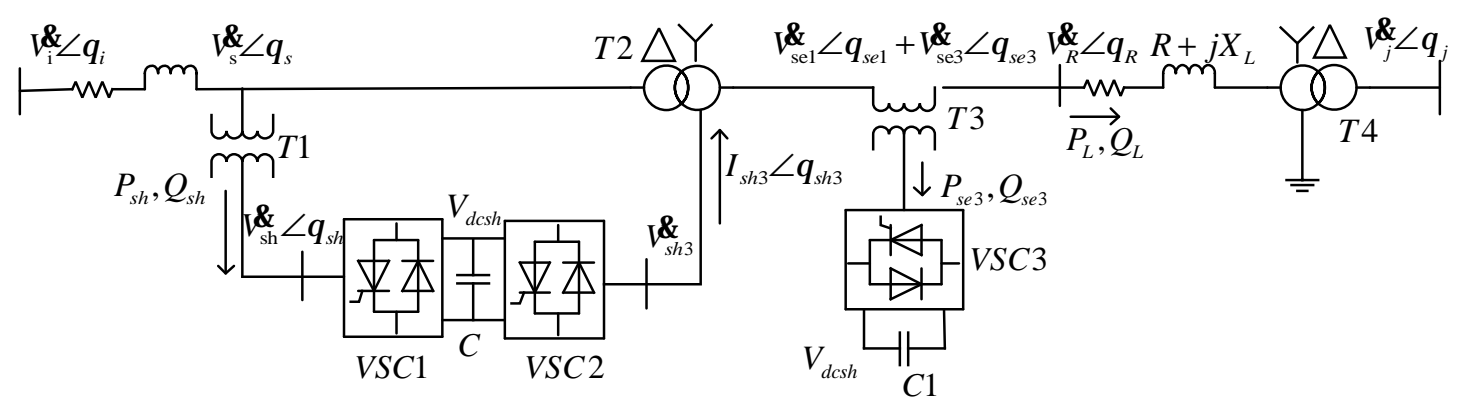

Figure 1 DPFC simplified schematic diagram of the simplified schematic

As shown in Figure 1, the parallel side is composed of two converters. The VSC1 is a three-phase converter and the VSC2 is a single phase converter. The two converters share a common DC capacitor. The power grid voltage joins into the AC side of the VSC1 through a parallel side coupling transformer T1, VSC1 absorbs the active power of the power grid to remain 
the DC capacitor voltage constant, converter VSC2 (end grounding) exports third harmonic of which the current distributes equably in the transmission lines through the neutral point of the $\mathrm{Y}$ side of the Y- $\Delta$ transformer T2. While the third harmonic current is cut off by the $\Delta$ side of the transformer and it can't feed into the transmission lines. Therefore, the transmission lines form into a loop through the grounding neutral point of the Y side of Y- $\Delta$ transformer T4 which is located on the end.

The series sides consist of many single-phase inverters. The single-phase converters are hanging in each phase of the transmission line through single box transformer T3. When it works independently, it is same to distributed static series compensator (DSSC). It can also work with parallel side as a result of the DPFC, by absorbing the harmonic power provided by the parallel side harmonic converter VSC2 transmission line, which can realize the exchange of power between series sides and parallel side. Because of the topology of DPFC, which can be used to implement a distributed power flow controller, it lays the foundation for the high reliability of the operation as the partial mechanism failure.

\section{DPFC location based on sensitivity factor [2]}

The performance of the application of DPFC in the power system is the adjustment of the line voltage, phase angle, compensation of the line impedance, dynamic control of the line power flow and reactive power compensation. Its advantages are flexible control, scattered layout, convenient installation and maintenance, but the most important issue of implementation are how to choose the DPFC best installation location and capacity, and how to improve the power system stability and security level to the maximum. Nowadays few literatures and projects related to the DPFC site selection and capacity determination, so determining the DPFC capacity and site selection method referring to UPFC.

\section{line active power flow performance index}

The severity of the power system load can be expressed by the line active power flow performance index [3], which is defined as follows:

$$
P I=\sum_{m=1}^{N_{1}} \frac{w_{m}}{2 n}\left(\frac{P_{l m}}{P_{l m}^{\max }}\right)^{2 n}
$$

Type $P_{1}^{\mathrm{m}}$ is the branch power flow, $P_{\mathrm{lm}}{ }^{\max }$ is a branch of rated active power, $\mathrm{n}$ is index coefficient, $\mathrm{W}_{\mathrm{m}}$ is weight coefficient that reflects the importance of branch, $N_{1}$ is the number of branch of the system. The PI parameters are defined in this way, it can intuitively reflect the actual trend and the trend limit between the limit, including transmission line congestion information system.

\section{DPFC power flow sensitivity factor [4]}

Solving the partial derivatives of two variables: series injected voltage magnitude and phase angle $\left(V_{\mathrm{S}}, \varphi_{\mathrm{S}}\right)$ by PI in order to achieve two control sensitivity. Through the sensitivity accurately determining the installation location of the parallel side of DPFC. When the branch $K$ is equipped with DPFC, the sensitivity coefficients of the line active power flow performing to the control index $X_{\mathrm{k}}\left(V_{\mathrm{S}}, \varphi_{\mathrm{S}}\right)$ are:

$$
\begin{aligned}
& c_{1}^{k}=\left.\frac{\partial P I}{\partial V_{s}}\right|_{V_{s}=0} \\
& c_{2}^{k}=\left.\frac{\partial P I}{V_{s} \partial \varphi_{s}}\right|_{\varphi_{s}=0}
\end{aligned}
$$


In order to eliminate the influence of the interaction between the two control variables $\left(V_{\mathrm{S}}, \varphi_{\mathrm{S}}\right)$, the mathematical theory can be known that the following method can be achieved. Namely: take the value of PI for the partial derivation of $V_{\mathrm{S}}=0$ as the value of $c_{1}{ }^{\mathrm{k}}$, at this time take the $\varphi_{\mathrm{S}}=0$. For the PI of the partial derivative of the $\varphi_{\mathrm{S}}=0$, meanwhile, divided by the value of the value $V_{\mathrm{S}}$ as the value $c_{2}{ }^{\mathrm{k}}$.The magnitude of the type $c_{1}{ }^{\mathrm{k}}, c_{2}{ }^{\mathrm{k}}$ are corresponding to the effective performance index of the line active power flow regulation of the two control variables of DPFC respectively.

\section{DPFC location calculation process}

By the definition of PI, we can get:

$$
\frac{\partial P I}{\partial X_{k}}=\sum_{m=1}^{N_{l}} w_{m} P_{l m}^{2 n-1}\left(\frac{1}{P_{l m}^{\max }}\right)^{2 n} \frac{\partial P_{l m}}{\partial X_{k}}
$$

The variation of the line power flow causing by the node injection power changes is defined by nodal injection power transferring distribution factor:

$$
\frac{\partial P_{l m}}{\partial X_{k}}=\frac{\partial P_{l m}}{\partial P_{i}} \bullet \frac{\partial P_{i}}{\partial X_{k}}+\frac{\partial P_{l m}}{\partial P_{j}} \bullet \frac{\partial P_{j}}{\partial X_{k}}
$$

The influence coefficient matrix of active power flow to the branch power flow is obtained by using the DC power flow calculation:

$$
\begin{aligned}
\Delta \theta & =X P \\
P_{i j} & =\frac{\theta_{i}-\theta_{j}}{x_{i j}}
\end{aligned}
$$

The coefficient matrix of the branch m (node $\mathrm{i}$ flow to node $\mathrm{j}$ ) is influenced by the active power flow $P_{\mathrm{n}}$ in the node $\mathrm{n}$.

$$
S_{m n}=\frac{d}{d P_{n}}\left(\frac{\theta_{i}-\theta_{j}}{x_{i j}}\right)=\frac{1}{x_{l m}}\left(\frac{d \theta_{i}}{d P_{n}}-\frac{d \theta_{j}}{d P_{n}}\right)=\frac{x_{i n}-x_{j n}}{x_{l m}}
$$

Among them, $x_{1}^{\mathrm{m}}$ is for the reactance of the branch $\mathrm{m}, P_{\mathrm{n}}$ is the injected power of the node, $x_{\mathrm{in}}$ and $x_{\mathrm{jn}}$ are the elements in the column $\mathrm{n}$ and column $\mathrm{n}$ of the first I column of the matrix X.

When the branch $\mathrm{m}$ is installed on the line (i.e. $\mathrm{m}=\mathrm{k}$ ):

$$
P_{l m}=\sum_{\substack{n=1 \\ n \neq s}}^{N_{\varphi}} S_{m n} P_{n}+P_{j s}
$$

When the branch $\mathrm{m}$ is not installed on the line (that is, $\mathrm{k} \mathrm{m}$ ):

$P_{l m}=\sum_{\substack{n=1 \\ n \neq s}}^{N_{1}} S_{m n} P_{n}$

In the formula: the total number of the system bus, the node $S$ is the balance node.

Therefore: When $\mathrm{m}=\mathrm{k}$,

$\frac{\partial P_{l m}}{\partial X_{k}}=S_{m i} \bullet \frac{\partial P_{i s}}{\partial X_{k}}+S_{m j} \bullet \frac{\partial P_{j s}}{\partial X_{k}}+\frac{\partial P_{j s}}{\partial X_{k}}$

When $\mathrm{m} \neq \mathrm{k}$, 
$\frac{\partial P_{l m}}{\partial X_{k}}=S_{m i} \bullet \frac{\partial P_{i s}}{\partial X_{k}}+S_{m j} \bullet \frac{\partial P_{j s}}{\partial X_{k}}=0$

According to the calculation model of power injection model [6]:

$$
\begin{gathered}
P_{i s}=-V_{s}^{2} g_{i j}-2 V_{i} V_{s} g_{i j} \cos \left(\varphi_{s}-\delta_{i}\right)+V_{j} V_{s}\left[g_{i j} \cos \left(\varphi_{s}-\delta_{j}\right)+b_{i j} \sin \left(\varphi_{s}-\delta_{j}\right)\right] \\
Q_{i s}=V_{i} I_{q}+V_{i} V_{s}\left[g_{i j} \sin \left(\varphi_{s}-\delta_{i}\right)+\left(b_{i j}+B / 2\right) \cos \left(\varphi_{S}-\delta_{i}\right)\right]
\end{gathered}
$$

Above (13) (14) formula:

$$
\begin{aligned}
& \left.\frac{\partial P_{i s}}{\partial V_{s}}\right|_{V_{s}=0}=-2 V_{i} g_{i j} \cos \left(\varphi_{s}-\delta_{i}\right)+V_{j}\left[g_{i j} \cos \left(\varphi_{s}-\delta_{j}\right)+b_{i j} \sin \left(\varphi_{s}-\delta_{j}\right)\right] \\
& \left.\frac{\partial P_{i s}}{V_{s} \partial \varphi_{s}}\right|_{\varphi_{s}=0}=-2 V_{i} g_{i j} \sin \delta_{i}+V_{j}\left(g_{i j} \sin \delta_{j}+b_{i j} \cos \delta_{j}\right) \\
& \left.\frac{\partial P_{j s}}{\partial V_{s}}\right|_{V_{s}=0}=V_{j}\left[g_{i j} \cos \left(\varphi_{s}-\delta_{i}\right)-b_{i j} \sin \left(\varphi_{s}-\delta_{j}\right)\right] \\
& \left.\frac{\partial P_{j s}}{V_{s} \partial \varphi_{s}}\right|_{\varphi_{s}=0}=V_{j}\left(g_{i j} \sin \delta_{j}-b_{i j} \cos \delta_{j}\right)
\end{aligned}
$$

Simultaneous equations (2)-(3), (11)-(16) can be calculated separately $c_{1}{ }^{\mathrm{k}}$ and $c_{2}{ }^{\mathrm{k}}$.

DPFC location method refer to the UPFC location selection method which demonstrate above, and we can get the best installation address of the DPFC parallel side which we need, and the series side is distributed in the line. Because the DPFC device has the characteristics of dispersion and small capacity so that the UPFC location method can only be used for reference. The specific installation location should be calculated according to the actual situation and the selection of capacity.

\section{DPFC capacity study}

\section{DPFC constant volume equivalent circuit [2]}

In the steady state, the DPFC can be expressed by the equivalent voltage source, the DPFC constant capacitance equivalent circuit is shown in Figure 2, and the resistance of the transmission line and the capacitance to ground are neglected.

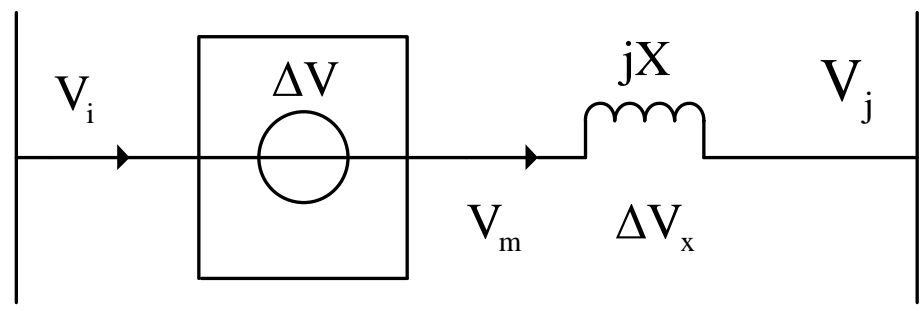

Fig. 2 DPFC constant volume equivalent circuit

DPFC steady-state operation of the phasor diagram [3] as shown in figure 3: 


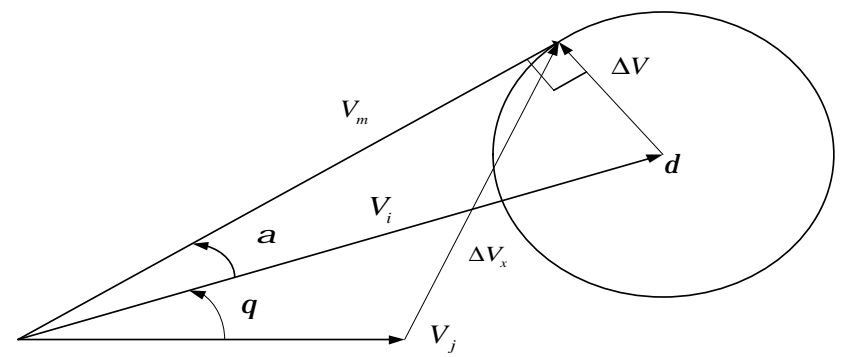

Fig. 3 the phasor diagram of the steady state operation of the DPFC

Referring to UPFC, when the DPFC is not installed in the transmission circuit, the active power flow over the line is:

$$
P_{d 0}=\frac{V_{i} \bullet V_{j}}{X} \sin \theta
$$

If the line is installed with DPFC, when the maximum $\alpha$, that is, $\Delta \mathrm{V}$ is with the vertical $V_{\mathrm{m}}$, making the line trend is the largest, at this time the flow of active power is:

$$
P_{d}=\frac{V_{m} \bullet V_{j}}{X} \sin (\theta+\alpha)
$$

Becauce $\sin \alpha=\Delta V / V_{\mathrm{m}}, \cos \alpha=V_{\mathrm{m}} / V_{\mathrm{i}} \approx 1$,

And because of the installation of the DPFC, so the line on the active power flow

$\Delta P=P_{d}-P_{d o}=\frac{V_{i} \bullet V_{j}}{X}\left(\cos \theta \frac{\Delta V}{V_{i}}-\sin \theta \frac{\Delta V^{2}}{V_{i}^{2}}\right)$

From the equivalent circuit of DPFC, $V_{\mathrm{m}}=V_{\mathrm{j}}+\Delta V_{\mathrm{x}}$, it is known that the cosine theorem:

$\Delta V_{x}=\sqrt{V_{m}^{2}+V_{j}^{2}-2 V_{j} V_{m} \cos (\alpha+\theta)} \approx \sqrt{\left|V_{i}^{2}-\Delta V^{2}+V_{j}^{2}-2 V_{j}\left(V_{i} \cos \theta-\Delta V \sin \theta\right)\right|}$

Becauce $I_{\mathrm{x}}=\Delta V_{\mathrm{x}} / \mathrm{X}$, so the running capacity of DPFC is:

$$
S=I_{x} \bullet \Delta V=\Delta V \bullet \frac{\sqrt{\left|V_{i}^{2}-\Delta V^{2}+V_{j}^{2}-2 V_{j}\left(V_{i} \cos \theta-\Delta V \sin \theta\right)\right|}}{X}
$$

This method is used to determine the capacity of DPFC. After solving the capacitance equivalent circuit, the DPFC investment cost function and profit function should be calculated so that the mathematical model of the optimal capacity of DPFC can be achieved. Solving the equations to determine the DPFC capacity. In fact, location is the first element to consider, then ensure the capacity.

\section{DPFC investment cost function}

According to the formula given by [5], the investment cost function of DPFC can be obtained.

$$
\begin{aligned}
& C_{1}=1000 C_{u p f c}^{\prime} \times S \\
& C_{d p f c}^{\prime}=0.0003 S^{2}-0.2691 S+188.22
\end{aligned}
$$

In the formula $\mathrm{S}$ is DPFC's capacity (MVA), the unit of $C_{\mathrm{DPFC}}$ is US\$/KVA. $C_{\mathrm{DPFC}}$ is the investment cost per unit capacity for DPFC.

\section{DPFC revenue function}

According to the principle that the transmission capacity of the power system is improved, the mathematical model is established according to the income function which is brought by the 
improvement of the transmission capacity:

$$
C_{2}=C_{2 a}+C_{2 b}
$$

In the above formula, the revenue brought by the increase in transmission capacity is related to the value of the income and the increase of the active power in different price. Expression for:

$$
C_{2 a}=t(p)\left(P_{\mathrm{d}}-P_{d 0}\right)
$$

Among them, the unit of $C_{2 \mathrm{a}}$ is US\$/year, $\mathrm{t}_{(\mathrm{P})}$ is the price coefficient and $P_{\mathrm{d}}$ is the maximum transmission capacity of a system which is installed with DPFC, $P_{\mathrm{d} 0}$ is the largest transmission capacity of a system without DPFC. The reference [2] is given at the annual price earnings, as shown in figure 4:

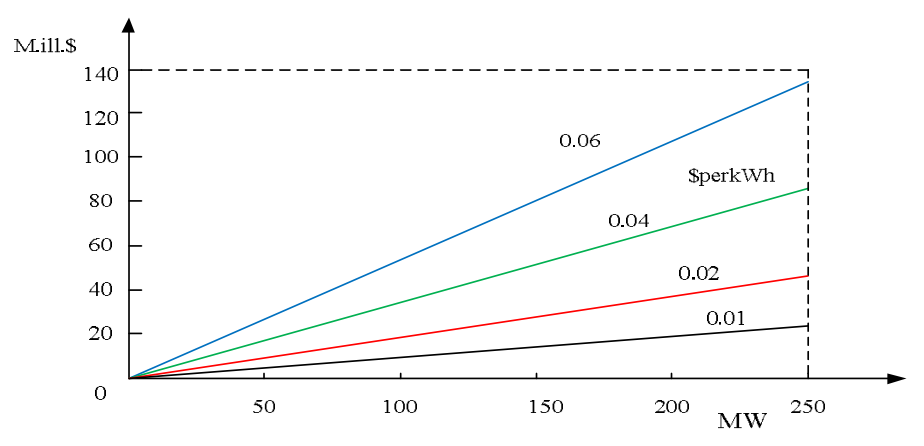

Fig. 4 annual income corresponding to different electricity price

$C_{2 \mathrm{~b}}$ represent the profit which is caused by the installation of a DPFC to avoid the construction of new transmission lines and systems brought about by the benefits. Its expression is:

$$
C_{2 b}=r \bullet C_{L}
$$

In the formula, the unit of $\mathrm{C}_{2 \mathrm{~b}}$ is US $\$, \mathrm{r}$ is electric power industry investment recovery rate, $C_{\mathrm{L}}$ is the cost of investment in the construction of new transmission lines which is used to establish new transmission for meeting power system demands according to the actual situation without FACTS. Reference [2] gives a one-time investment cost corresponding to different line lengths of different voltage levels, as shown in Figure 5.

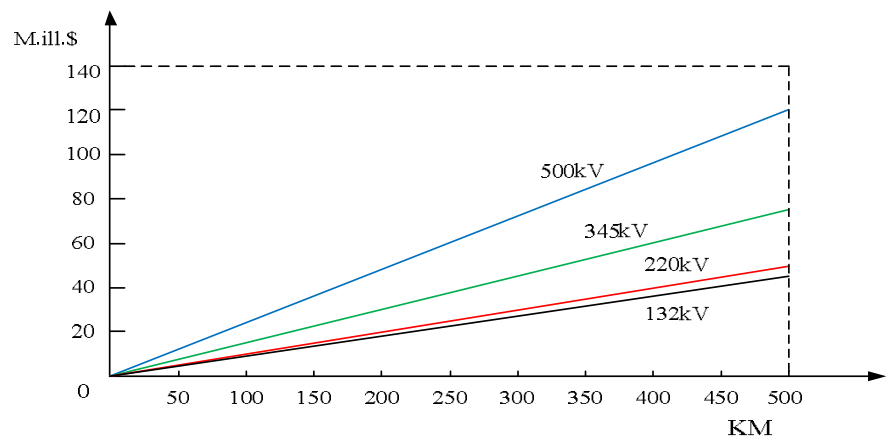

Figure 5 the relationship between the length of the new transmission line and the cost of the investment

From the formula (4.9) and formula (4.10) we can know that the income function $C_{2}$ of the unit is US\$/year, and the unit of the investment cost function $C_{1}$ is US\$, the two units are different, so carry $C_{1}$ on the conversion:

$$
C_{1}^{\prime}=\alpha \bullet C_{1}
$$




$$
\alpha=\frac{r(1+r)^{n}}{(1+r)^{n}-1}
$$

In the formula, $\mathrm{n}$ is DPFC equipment economic life, $\alpha$ is the present value of coefficient for the next year, $\mathrm{r}$ is the investment recovery rate of electric power industry. Converting $\mathrm{C}_{1}$ to annual investment costs $\mathrm{C}_{1}{ }^{\prime}$, and thus consistent with the unit $\mathrm{C}_{2}$.

\section{Mathematical model of the optimal capacity of DPFC}

To sum up, the optimal capacity of DPFC mathematical relationship is as follows:

$$
\max : C_{\text {total }}=C_{2}-C_{1}^{\prime}
$$

The mathematical relationship of the optimal capacity of DPFC is the target function. The $C_{\text {total }}$ is the total income of DPFC, according to the maximum of which can we determine the capacity of the DPFC. This needs to optimize the objective function. Looking for the optimization method, this paper will use the Matlab computing function for the maximum value of the objective function optimization.

\section{Conclusions}

This paper introduces a location selecting and capacity determining method of the DPFC. The location selection of DPFC based on the sensitivity factor, and the capacity determination in economic conditions of DPFC pave the way for practical project apply and for the future study of the properties of the DPFC. But in the practical application of DPFC, there may be a variety of problems of other aspects, the method of location selection and setting needs to be adjusted appropriately.

\section{References}

[1] Zhihui Yuan, Sjoerd W.H. de Haan, Braham Ferreira. A FACTS Device-Distributed Power Flow Controller (DPFC) [J]. Power Electronics, IEEE Transactions on, 2010: 2564-2572.

[2] Xin Liu. Application Research and Modeling of unified power flow controller (UPFC) [D]. North China Electric Power University (Beijing), 2009.

[3] Prabha Kundur, John Paserba, Venkat Ajjarapu, et al. Definition and Classification of Power System Stability. IEEE Transactions on Power Systems. 2004, 19(2): 1387-1393.

[4] M. Saravanan, etal, Application of PSO technique for optimal location of FACTS devices considering system loadability and cost of installation, Power Engineering Conferenee,Vol.2 pp.716-721, 29 Nov.2 Dec.2005

[5] Yulin Shi. Design of UPFC multi objective controller and study of its influence on power system [D]. Nanjing: Nanjing University of Science and Technology, 2010.

[6] Qiang Tong. Study on power flow calculation model and algorithm for power system with FACTS [D]. Hunan University, 2005. 